\title{
Politische Erwachsenenbildung
}

\author{
Ewa Przybylska
}

Online publiziert: 27. Juni 2015

(c) Die Autor(en) 2015. Dieser Artikel ist auf Springerlink.com mit Open Access verfügbar.

\section{Rezension zu}

Tetyana Kloubert (2014). Aufarbeitung der Vergangenheit als Dimension der Erwachsenenbildung. Polnische, russländische und ukrainische Perspektive. Peter Lang Edition, Frankfurt a. M., 719 Seiten, $107 €$, ISBN 978-3-631-65101-8

Die vorliegende Studie ist ein attraktives Angebot für Forscher, die sich mit der Vergleichenden Erziehungswissenschaft beschäftigen. Das methodologische Selbstverständnis dieser wissenschaftlichen Disziplin befindet sich gegenwärtig immer noch in der Phase seiner Herausbildung. Die Disziplin bietet Platz sowohl für Anhänger methodologischer Strenge und quantitativer Methoden in der Empirie als auch für diejenigen, die sich stärker an jener humanistischen und intuitiven Ausrichtung orientieren, welche sich auf historische und philosophische Forschungen stützt.

Wo ist die rezensierte Publikation zu verorten? Diese Vergleichsstudie ist ein einzigartiges Beispiel für die Verknüpfung der empirischen Methode mit Intuition, Reflexion und persönlichem Urteil auf der Basis einer profunden Kenntnis von Kulturen und Bildungssystemen in anderen Ländern: Die Autorin hat 91 Interviews mit Experten der politischen Bildung aus Polen, Russland und der Ukraine durchgeführt und im Sinne der Grounded Theory, nach welcher die Interviews thematisch (Codings) eingeordnet sind, ausgewertet. Zum einen versucht die Studie, den ,deutschen Diskurs durch die Perspektive auf die Erinnerungslandschaft der drei ost- und mitteleuropäischen Länder (Polen, Russland und der Ukraine) zu erweitern“ (S. 2). Zum anderen geht es darum, ,die ost- und mitteleuropäische Auseinandersetzung mit der Vergangenheit in Deutschland verständlicher zu machen“" (S. 10).

Prof. Dr. hab. E. Przybylska $(\bowtie)$

Nikolaus Kopernikus Universität in Toruń,

ul. Gagarina 9,

87-100 Toruń, Polen

E-Mail: evaprzyb@umk.pl 
Angesichts der beeindruckenden Materialfülle dürfte jede Bemerkung, die auf Fehlendes hinweist, unangebracht erscheinen; aber - bei allem Respekt für das Untersuchungskonzept - wäre es doch wünschenswert gewesen, auch ostdeutsche Perspektiven aufzunehmen. Damit wäre das ganze Werk vollständiger und hätte eine erhöhte Nützlichkeit. Eines der Hauptziele der Vergleichenden Erziehungswissenschaft ist doch gerade, ein besseres Verständnis vom eigenen Bildungssystem, von der Struktur und Praxis der Bildung, Kultur und Tradition sowie von deren sozialem Hintergrund zu schaffen.

Ein unbestreitbarer Vorteil der Studie ist ihre transparente und klare Struktur. Die „Theoretische Einführung“ liefert einen breiten Einblick in den aktuellen Forschungsstand zur Vergangenheitsbewältigung auf historischer, politischer, rechtlicher, künstlerisch-gestaltender, gesellschaftlicher, anthropologisch-philosophischer und pädagogischer Ebene. Im nächsten Abschnitt erfolgen die Darstellung und Analyse der Daten aus drei Ländern. Der analoge Aufbau der Auswertung fördert das Verständnis und ermöglicht eine vergleichende Beobachtung. Die Hauptforschungsfragen beziehen sich hierbei auf die Aufarbeitung der Vergangenheit im Prozess der gesellschaftlichen Transformation, in Hinblick auf Demokratie, gesellschaftliche Integration und pädagogisches Selbstverständnis. Anschließend folgen ein Vergleich der Ergebnisse sowie eine Darstellung pädagogischer Konsequenzen und Empfehlungen.

Die Untersuchung erfüllt die an Vergleichsstudien gestellten Anforderungen; sie enthält eine Analyse der Wechselbeziehung zwischen Gesellschaft und Bildungssystem, nimmt Bezug auf interkulturelle Daten und zielt darauf ab, Regeln und Gesetzmäßigkeiten der untersuchten Phänomene zu entdecken. Die Autorin verweist auf die komplexen Beziehungen zwischen dem Bildungssystem und den Eigenschaften der Sozialstruktur und sensibilisiert den Leser für das Bedingungsgefüge, das auf Herangehensweisen bei der Aufarbeitung der Vergangenheit einwirkt.

Der kritische Punkt des Forschungsprojekts ist paradoxerweise die politische Erwachsenenbildung in den untersuchten Ländern. Die Autorin ist sich dieser Schwäche vollauf bewusst: „Die Maßnahmen der politischen Erwachsenenbildung haben nicht den gleichen Rang und die Verbreitung, wie es in Deutschland der Fall ist" (S. 155). Maßnahmen im Rahmen der politischen Bildung sind im Allgemeinen von sporadischem, chaotischem und amateurhaftem Charakter. Es gibt weder eine öffentliche Förderung politischer Bildung noch eine professionelle Qualifizierung der Erwachsenenbildner. Es kann daher nicht nur der Autorin angelastet werden, wenn in so manchem Interview unbelegbare Behauptungen auftauchen, wie etwa jene eines polnischen Erwachsenenbildners: ,Vom alten System ist bei uns die Überzeugung geblieben, dass nur formelle Bildung einen Sinn hat" (S. 233). Aus solcherlei Aussagen können keine verallgemeinerbaren Rückschlüsse gezogen werden. Einige ernsthafte Studien zum Thema Bildung, insbesondere Erwachsenenbildung, auf deren Grundlage Interviewaussagen hätten hinterfragt oder überprüft werden können, finden sich nicht in der Literaturliste.

Kann man die Meinungen der Befragten als repräsentativ für die Erwachsenenbildung in Betracht ziehen oder sind sie eher als Ausdruck persönlicher Involviertheit der Erwachsenenbildner in ihrem Umgang mit der Vergangenheit zu betrachten? Ist 
es überhaupt möglich, im Falle Polens, Russlands und der Ukraine von Transformationsgesellschaften zu sprechen?

Im Buch gibt es viele umstrittene Thesen. Ein Grund mehr, es zu lesen. Ich empfehle es allen, die sich für die Geschichte und das gesellschaftspolitische Leben in Mittel- und Osteuropa interessieren, und vor allem Liebhabern der international vergleichenden Forschung zur Erwachsenenbildung.

Open Access Dieser Artikel wird unter der Creative Commons Namensnennung 4.0 International Lizenz (http://creativecommons.org/licenses/by/4.0/deed.de) veröffentlicht, welche die uneingeschränkte Nutzung, Verbreitung und Wiedergabe für beliebige Zwecke erlaubt, sofern Sie den/die ursprünglichen Autor(en) und die Quelle ordnungsgemäß nennen, einen Link zur Creative Commons Lizenz beifügen und angeben, ob Änderungen vorgenommen wurden. 